e-J. Surf. Sci. Nanotech. Vol. 3 (2005) 79-81

Conference - Nano-org. \& Func. -

\title{
Ultra-small chamber for single-molecule detection of biological reaction*
}

\author{
Hiroyuki Noji ${ }^{\dagger}$ \\ Institute of Industrial Science, University of Tokyo, \\ 4-6-1 Komaba Meguro-ku, Tokyo 153-8505, Japan \\ Yannick Rondelez \\ LIMMS/IIS/CNRS, University of Tokyo, 4-6-1 Komaba Meguro-ku, Tokyo 153-8505, Japan \\ Takako Nakashima \\ Institute of Industrial Science, University of Tokyo, \\ 4-6-1 Komaba Meguro-ku, Tokyo 153-8505, Japan, \\ Guillaume Tresset \\ LIMMS/IIS/CNRS, University of Tokyo, 4-6-1 Komaba Meguro-ku, Tokyo 153-8505, Japan, \\ Kazuhito Tabata \\ Institute of Industrial Science, University of Tokyo, \\ 4-6-1 Komaba Meguro-ku, Tokyo 153-8505, Japan. \\ Yasuyuki Kato-Yamada \\ Collge of Science, Rikkyo (St Paul's) University, \\ 3-34-1 Nishi-Ikebukuro, Toshima-ku, Tokyo 171-8501, Japan \\ Hiroyuki Fujita and Shoji Takeuchi \\ Center for International Research on Micromechatronics (CIRMM), \\ Institute of Industrial Science, University of Tokyo, \\ 4-6-1 Komaba, Meguro-ku, Tokyo 153-8505, Japan \\ (Received 2 November 2004; Accepted 1 February 2005; Published 1 March 2005)
}

\begin{abstract}
We report here development of ultra-small reaction chamber array from silicone-elastomer sheet and glass plate, and its applications for biological assay. It was shown that single-molecules of protein, or DNA can be enclosed in each hermetic chamber stably. The volumes of chambers are extremely small ( $6 \sim 10$ femtoliters) so that small numbers of molecule $(\sim 2000)$ can reach to detectable concentrations ( micro molars). When single enzyme molecules were enclosed in the chambers, its catalytic activity was fluorescently monitored to demonstrate the feasibility of single-molecule assay using the chamber. We also apply this method to access reaction efficiency of protein molecular motor, $\mathrm{F}_{1}$-ATPase. Motor molecules were enclosed and manipulated to rotate reversely using magnetic tweezers. The resultant extremely small amount of synthesized ATP was calculated from the speed of the motor after released from the manipulation. This measurement revealed first time that the motor is designed to tightly couple the chemical reaction and mechanical event. Thus, it was proven that the ultra-small chamber can be a new strong tool for ultra-sensitive bioassay. [DOI: 10.1380/ejssnt.2005.79]
\end{abstract}

Keywords: Ultra-small reaction chamber; Biological molecules-nucleic acids; Enzyme; Single-molecule assay; Molecular motor

\section{INTRODUCTION}

Single-molecule detection and analysis has been achieved mainly with elaborated optical microscopic techniques which limit observation volumes to reduce background noise. An alternate approach is physical confinement of a biomolecule in an extremely small volume like some femotoliters. In such a small volume, even for a single molecule of conventional enzymes with a turn-over rate of $10 / \mathrm{sec}$, it is possible to accumulate catalytic product molecules up to $\mu \mathrm{Ms}$ within a minute. Simple reduction of working volume should enable single-molecule detection of biomolecules. However, one would be faced with mainly three difficulties for down-sizing of working volume:

( i ) quick evaporation especially for the nanoliter level, ( ii ) precise droplet positioning,

( iii ) precise control of the volume and monodispersity.

Here, we report the development of the smallest reaction chamber array from a silicone elastomer, PDMS, using conventional microfabrication techniques and demonstrate single-enzyme assays using conventional fluorescent reaction kits. We also challenged to reveal a fundamental coupling efficiency of a molecular motor, F1-ATPase using this technique.

\section{EXPERIMENTS}

Microfabricated PDMS sheet was prepared as described (Fig.1(A)) [1]. A 1.5- $\mu$ m-thick SOI wafer was covered
*This paper was presented at International Symposium on Nanoorganization and Function, Tokyo, Japan, 11-12 November, 2004.

${ }^{\dagger}$ Corresponding author: hnnoji@iis.u-tokyo.ac.jp 
(A)

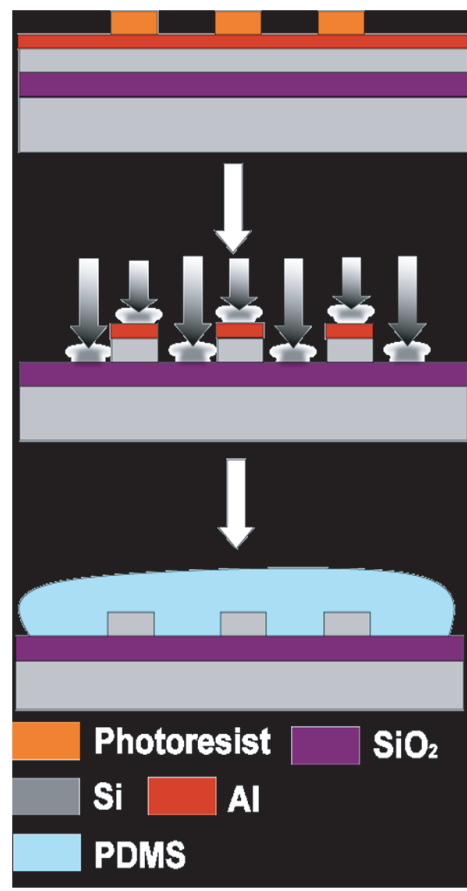

(B)

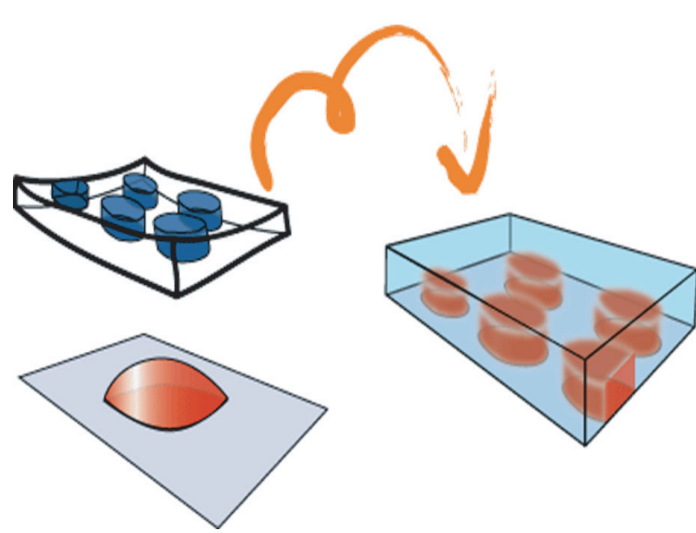

FIG. 1: Schematic image of microfabrication procedures of micro-chambers (A), and preparation of enclosure of solutions in chambers (B).

with an aluminum mask, and, then, the mask was patterned by photolithography. After etching and removing the aluminum layer, regular arrays of identical cylindrical shapes were prepared on the SOI wafer. Liquid PDMS was poured on the wafer, cured at $90^{\circ} \mathrm{C}$ for polymerization, and peeled off. Mold patterns were precisely reproduced.

Buffer solution was introduced into the microchambers by sandwiching a droplet between a microscope slide and the patterned PDMS sheet. Resultantly, solution was homogeneously distributed by capillarity. By pressing, gas bubbles and excess water were evacuated, and then the PDMS sheet bond to the glass surface through van-derWaals force. This step distributes the buffer among up to $10^{5}$ independent reaction vessels per $\mathrm{mm}^{2}$, each of them containing a few femtoliters of liquid solution (Fig. 1(B)).

In the case of enzymes, the microarray allows to trap both the proteins and their own substrate/products in the same chamber. Because of the extremely small volume, a minute quantity of enzymatic products is enough to reach a detectable concentration, and we can therefore expect to detect directly the individual activities of isolated single enzymes. We have demonstrated this idea using $\beta$-Galactosidase ( $\beta$-Gal) or Horseradish Peroxidase (HRP) as model enzymes. A fluorogenic commercial activity assay mixture was distributed over the whole array of $30 \mathrm{fL}$ containers. After closing, the fluorescent intensity was then measured simultaneously in approximately 300 chambers, using a microscope. When the enzyme solution was diluted down to a ratio close to 1.7 enzyme molecules per chamber, it appeared that the brightness in the array was not homogeneously distributed (Fig. 2). More precisely, the intensity was quantized among a few discrete

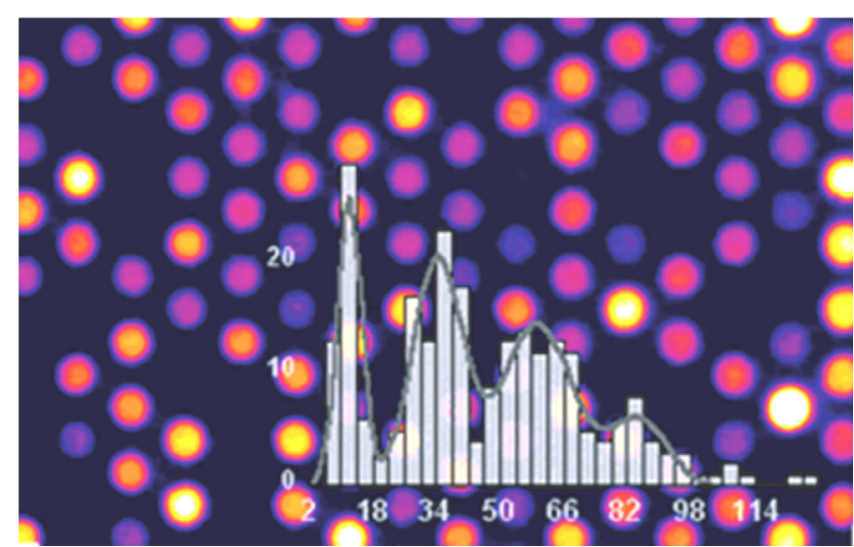

FIG. 2: Fluorescent image of single molecules enzymatic activities of $\beta$-galactosidase.

levels: dark, medium and bright chambers were easily distinguished. Probabilities of chamber for each fluorescent level are roughly consistent with the expected values for 0 , 1, 2, 3 enzyme per chamber $(31,26$, and $15 \%$ ) according to the Poisson distribution. Thus, single-enzyme detection was achieved using simple logic and experimental set-up.

We then turned to the more challenging experiment of a molecular motor protein, F1-motor. This motor is a rotary motor protein, which makes the rotation of the central rotary shaft powered by the ATP-hydrolyzing stator part. It is expected that F1-motor synthesizes ATP molecules when it is enforced to rotate in the reverse direction. However, the estimated number of synthesized ATP molecules is extremely small so that the coupling 
(A)

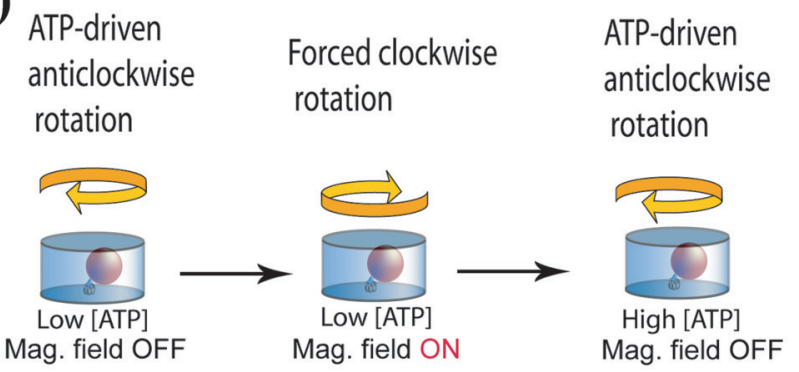

(C)

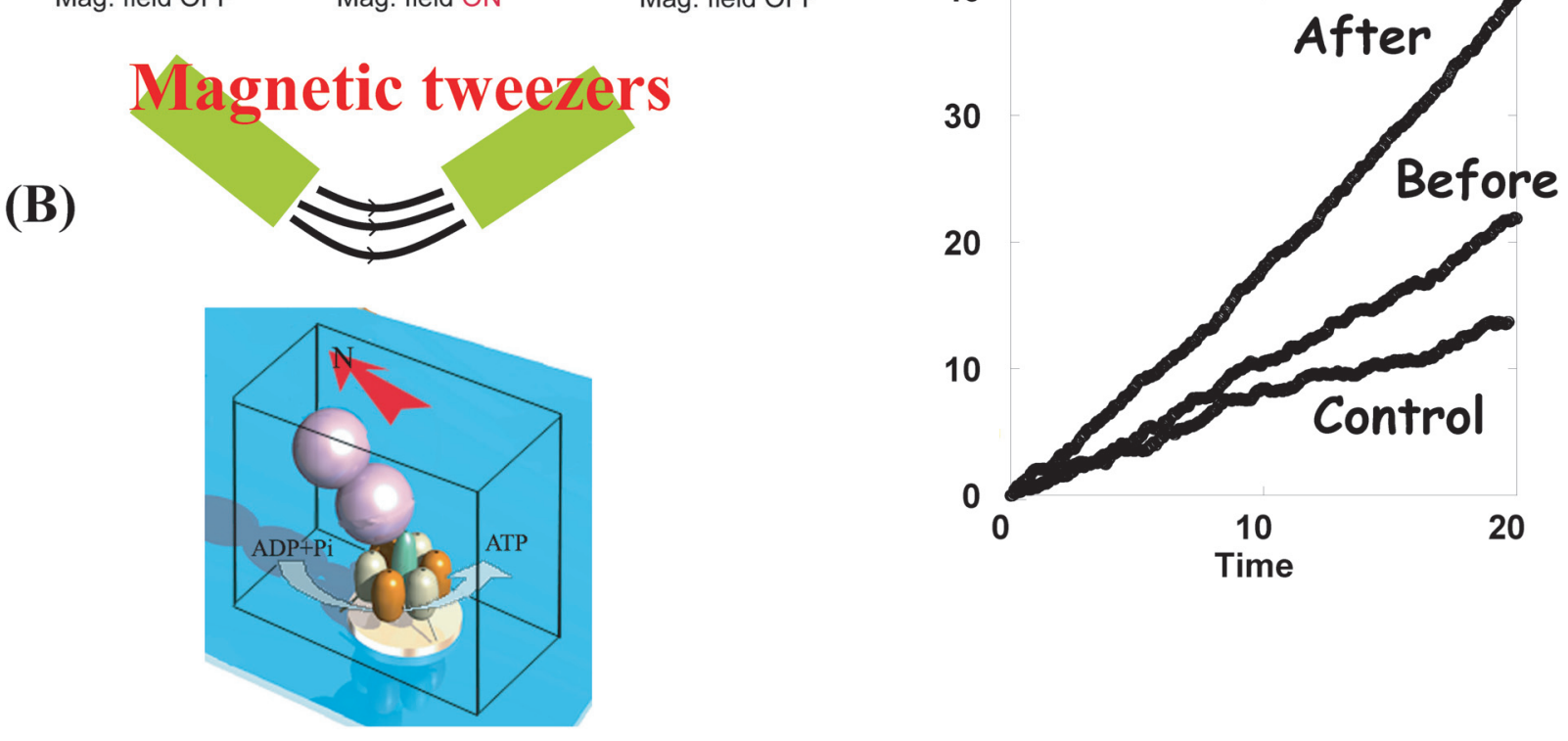

FIG. 3: Determination of mechanochemical coupling efficiency of F1 motor using a microchamber. Schematic image and experimental set-up were shown (A) and (B). A time-course of experiment was shown (C). After forced rotation and release from the external magnetic field, the F1 molecule showed faster rotation indicating the ATP synthesis in the microchamber.

efficiency has not been measured so far; when the motor is turned at $10 \mathrm{~Hz}$ for $1 \mathrm{~min}$, the estimated number is calculated to be only 1800 molecule supposing 3 ATP per turn. In order to detect such a tiny amount of chemical out put, single F1 molecules were enclosed in femtolitre-sized hermetic chambers, and rotated reversely using magnetic tweezers. After release of the magnetic field, the F1 molecules showed faster rotation, reporting the amount of synthesized ATP as their speed increase. The total amount of synthesis was determined form the increment of the rotary speed after the motor is released from external magnetic field. It was revealed that the motor is designed to couple the mechanical energy input into chemical out put with a high efficiency, reaching $100 \%$.
[1] H. Noji, R. Yasuda, M. Yoshida, and K. Kinosita, Jr, Direct observation of the rotation of F1-ATPase, Nature 386, 299 (1997).

[2] Y. Rondelez, T. Guillaume, K. Tabata, H. Arata, H. Fujita, S. Takeuchi, and H. Noji, Microfabricated arrays of femtoliter chambers allow single molecule enzymology, Na- ture Biotechnology, in press.

[3] Y. Rondelez, T. Guillaume, K. Nakashima, Y. KatoYamada, H. Fujita, S. Takeuchi, and H. Noji, Highly coupled ATP synthesis by single F1 molecule, Nature, in press. 